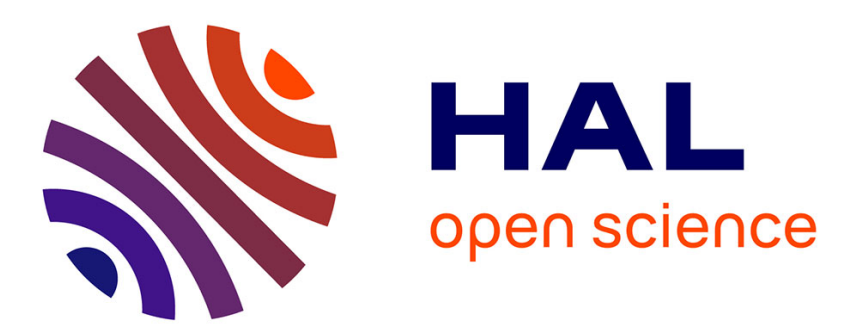

\title{
Microtexture de fibres de carbure de silicium. Etude par microscopie électronique par transmission \\ M. Guigon
}

\section{To cite this version:}

M. Guigon. Microtexture de fibres de carbure de silicium. Etude par microscopie électronique par transmission. Revue de Physique Appliquée, 1988, 23 (3), pp.229-238. 10.1051/rphysap:01988002303022900 . jpa-00245765

\section{HAL Id: jpa-00245765 https://hal.science/jpa-00245765}

Submitted on 1 Jan 1988

HAL is a multi-disciplinary open access archive for the deposit and dissemination of scientific research documents, whether they are published or not. The documents may come from teaching and research institutions in France or abroad, or from public or private research centers.
L'archive ouverte pluridisciplinaire HAL, est destinée au dépôt et à la diffusion de documents scientifiques de niveau recherche, publiés ou non, émanant des établissements d'enseignement et de recherche français ou étrangers, des laboratoires publics ou privés. 


\title{
Microtexture de fibres de carbure de silicium. Etude par microscopie électronique par transmission
}

\author{
M. Guigon \\ Division Polymères et Composites, Université de Technologie, 60200 Compiègne, France
}

(Reçu le 24 mars 1987, révisé le 9 juin 1987, accepté le 12 juin 1987)

\begin{abstract}
Résumé. - Les propriétés d'un matériau composite dépendent à la fois de celles du renfort, de la matrice et de l'interface. Afin de contribuer à la caractérisation des composites céramiques SiC-SiC, nous avons étudié la microtexture de fibres de carbure de silicium (Nicalon) par des techniques de microscopie électronique par transmission. Nous avons montré que toutes les fibres présentent une phase de carbure de silicium microcristallisée. La taille des domaines diffractants varie de 0,6 à $5 \mathrm{~nm}$ selon la fibre. Certaines fibres présentent des ségrégations de carbone soit amorphe, soit turbostratique.
\end{abstract}

\begin{abstract}
The properties of a composite material depend on fibres, matrix and interface properties. In order to study the ceramic composites SiC-SiC we characterized some commercial (Nicalon) silicon carbide fibres. Transmission electron microscopy was used. We showed that the fibres were made of a microcrystallised silicon carbide phase. Diffracting areas were observed of sizes varying from 0.6 to $5 \mathrm{~nm}$ according to the fibre. Some fibres showed amorphous or turbostratic carbon segregations.
\end{abstract}

\section{Introduction.}

Des études, effectuées sur les composites SiC-SiC, ont montré que les fibres de carbure de silicium n'ont pas le même comportement mécanique quand elles sont libres ou enchassées dans une matrice de carbure de silicium déposée sur la fibre en phase vapeur. Avant d'étudier ce phénomène « d'effet matrice ", il est évidemment nécessaire de connaître la microtexture des deux constituants : la fibre et la matrice. Dans ce travail, nous avons caractérisé des fibres SiC commerciales (Nicalon) identifiées par les appellations NLP 101, NLM 102, SiC 1000 et NLM 202.

\section{Techniques utilisées.}

Les techniques d'étude sont des techniques de microscopie électronique par transmission. Ces techniques sont les suivantes.

- Les techniques d'image en fond clair, utilisées pour étudier soit la morphologie des échantillons, soit la microtexture, à l'échelle des distances réticulaires, des microdomaines diffractants (franges de réseau).

- Les techniques de diffraction à aire sélectionnée.

REVUE DE PHYSIQUE APPLIQUÉE. - T. 23, N 3, MARS 1988
- Les techniques d'image en fond noir, qui consistent à faire l'image avec une partie du faisceau électronique diffracté par l'objet, sélectionnée par un diaphragme.

Techniques $d u$ fond noir.

Pour sélectionner la partie d'un faisceau diffusé, nous avons utilisé un diaphragme objectif dont l'ouverture correspond dans le plan d'Abbe à $2 \mathrm{~nm}^{-1}$.

Pour que le microscope restitue une image fidèle, il est alors nécessaire que le transfert de tous les faisceaux diffusés sélectionnés par le diaphragme objectif s'effectue sans déphasage parasite. Dans notre cas, ces conditions sont réalisées pour $\Delta f=$ $22 \mathrm{~nm}$ avec $C s=1,1 \mathrm{~mm}$ à $120 \mathrm{kV}$ ( $\Delta f$ est la défocalisation de l'objectif, $C s$ est la constante d'aberration sphérique du microscope).

La figure 1 représente à titre d'exemple les conditions expérimentales utilisées pour visualiser l'objet à l'aide du faisceau diffracté par la phase de carbure de silicium en position (111).

Nous avons étudié la nature cristalline ou amorphe des fibres en testant le caractère aléatoire ou non des images en fond noir $[1,2]$. En effet, si la structure est amorphe, l'image présente une granulométrie aléatoire dont la moyenne est donnée par la réponse 


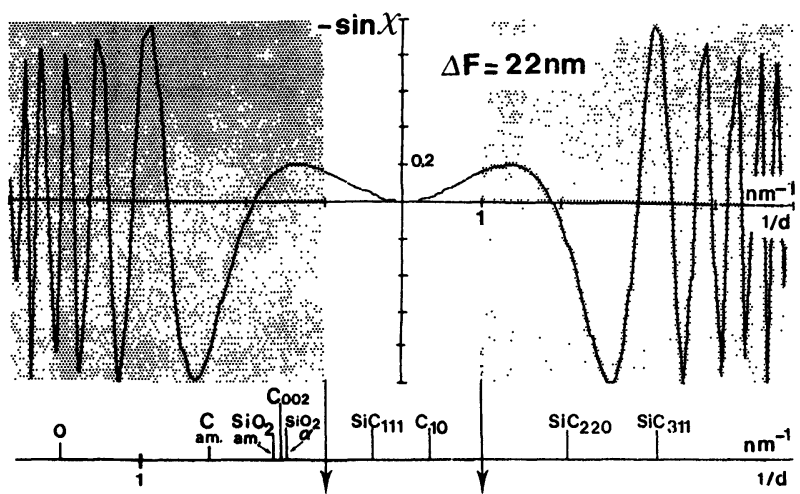

Fig. 1. - Représentation des conditions expérimentales utilisées pour identifier la phase de carbure de silicium $(\Delta F=22 \mathrm{~nm})$.

[Schematic representation of image conditions used to study the silicon carbide phase $(\Delta F=22 \mathrm{~nm})$.]

impulsionnelle de l'objectif, c'est-à-dire par la tache d'Airy (figure de diffraction) du diaphragme objectif qui nous permet de sélectionner une partie du faisceau diffusé. Pour une ouverture de $2 \mathrm{~nm}^{-1}$, la tache d'Airy est égale à $0,6 \mathrm{~nm}$. Afin de connaître si l'image présente des caractéristiques s'écartant de cette moyenne, donc à caractère non aléatoire, nous pouvons soit changer la taille du diaphragme objectif (si la taille diminue, la dimension des points augmente), soit changer la projection de l'échantillon. Toute région cristallisée ne se situant pas sur l'axe optique subit un déplacement de son image. On peut alors établir une corrélation entre deux images.

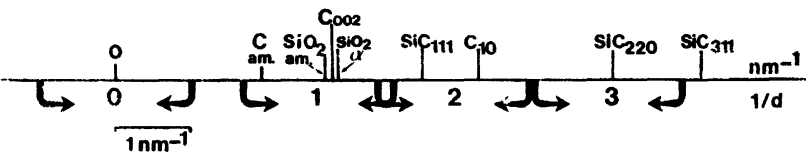

Fig. 2. - Représentation de l'emplacement théorique des faisceaux diffusés par une fibre de carbure de silicium.

[Representation of the theoretical position of diffuse beams by a silicon carbide fibre.]

Si nous considérons la figure 2 , nous remarquons qu'il est difficile de changer la taille du diaphragme objectif pour les raisons suivantes.

- Un diaphragme à ouverture plus grande laisse passer la diffusion inélastique des électrons en position 1. En outre, il ne permet pas de conserver le nombre de faisceaux sélectionnés si on tient compte de la largeur (non représentée) des faisceaux diffusés.

- Un diaphragme à ouverture plus petite impose un temps de pose plus long qui ne permet pas d'obtenir des images sans effet des instabilités mécaniques. De plus, pour une étude systématique des fibres, la tache d'Airy du diaphragme est trop importante par rapport à la taille des microdomaines (la tache d'Airy est de 1,2 nm pour une ouverture de $1 \mathrm{~nm}^{-1}$ ).

Ces raisons nous ont conduit à utiliser un diaphragme à ouverture de $2 \mathrm{~nm}^{-1}$ pour l'étude de la microtexture et à utiliser la technique de la défocalisation de l'objectif pour l'étude de la structure.

Il a fallu rechercher une valeur de la défocalisation suffisante pour détecter une translation de l'image et qui ne perturbe pas le transfert des ondes avec l'ouverture du diaphragme utilisé. La valeur optimale que l'on a trouvé est de $50 \mathrm{~nm}$. La fonction de transfert correspondante $(\Delta f=72 \mathrm{~nm})$ est représentée sur la figure 3 . Nous constatons que seuls les faisceaux situés dans une ouverture de $1,1 \mathrm{~nm}^{-1}$ sont transmis correctement. Ceci est satisfaisant pour notre étude. Par contre, une défocalisation de $100 \mathrm{~nm}$ ne convient pas car elle n'assure le transfert que dans une plage de $0,8 \mathrm{~nm}^{-1}$. Sur la figure 3 sont représentées à titre d'exemple les conditions expérimentales utilisées pour étudier la phase de carbure de silicium en position (111).

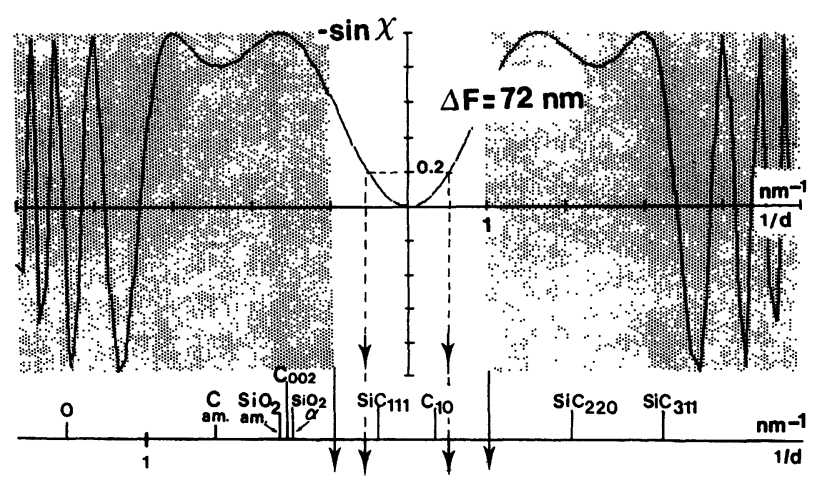

Fig. 3. - Représentation de la fonction de transfert, $\Delta f=72 \mathrm{~nm}$.

[Objective lens transfer function with $\Delta f=72 \mathrm{~nm}$.]

Ces conditions nous ont imposé des angles d'inclinaison du faisceau direct très précis pour chaque cas. Ils sont consignés dans le chapitre traitant des conditions expérimentales.

\section{Conditions expérimentales.}

Un certain nombre de travaux sur les fibres de carbure de silicium ont déjà été publiés [3-9]. Tous les auteurs ont montré que les fibres contiennent du silicium, du carbone et de l'oxygène. Selon ces auteurs, l'oxygène semble être consommé sous forme d'oxyde de silicium, tandis qu'une partie du carbone reste "libre». C'est à partir de ces données que nous avons tracé la figure 2. Elle représente l'emplacement théorique des faisceaux diffusés possibles dans le diagramme de diffraction d'une fibre SiC. 
On a :

- carbone C (amorphe : 0,53 nm, (10)),

- silice $\mathrm{SiO}_{2}$ (amorphe : 0,369 nm, (11)),

- carbone turbostratique $C(002 \geqslant 0,344 \mathrm{~nm})$,

- quartz $\alpha$ (101: 0,334 nm),

- carbure de silicium $\operatorname{SiC}_{\beta}(111: 0,251 \mathrm{~nm})$,

- carbure de silicium $\operatorname{SiC}_{\beta}(200: 0,217 \mathrm{~nm})$,

- carbone C (10:0,213 $\mathrm{nm})$,

- carbure de silicium $\operatorname{SiC}_{\beta}(220: 0,154 \mathrm{~nm})$,

- carbure de silicium $\operatorname{SiC}_{\beta}(311: 0,131 \mathrm{~nm})$,

- carbone C (11:0,123 nm).

Les positions $0,1,2,3$ représentent celles du diaphragme objectif. On peut faire soit une image en fond clair (position 0 ), soit des images en fond noir (positions 1, 2, 3). La position 1 permet de sélectionner $\mathrm{SiO}_{2}$ amorphe, $\mathrm{SiO}_{2} \alpha, \mathrm{C}$ amorphe et $\mathrm{C}$ turbostratique (002), la position 2 permet de sélectionner $\mathrm{SiC}_{\beta}$ (111) et $\mathrm{C}$ turbostratique (10) ; enfin, la position 3 sélectionne $\mathrm{SiC}_{\beta}$ (220).

Les angles d'inclinaison du faisceau direct sont les suivants :

- position $1 \rightarrow 9 \times 10^{-3} \mathrm{rad}$

- position $2 \rightarrow 15 \times 10^{-3} \mathrm{rad}$

- position $3 \rightarrow 22 \times 10^{-3} \mathrm{rad}$.

C'est à partir de ces différentes imageries que nous avons étudié la microtexture des fibres de carbure de silicium.

\section{Techniques de préparation.}

Tous les échantillons sont préparés par microtomie. Ils sont au préalable enrobés dans une résine époxyde puis coupés par ultramicrotomie à l'aide d'un couteau de diamant.

\section{Résultats.}

5.1 ETUde DE LA FIBRE NLM 102. - Cette fibre est caractérisée par de fréquentes ségrégations en bord de fragment (Fig. 4) visualisées en position 1 (Fig. 2). Ces ségrégations sont formées de points lumineux de dimension quasi constante $(0,6-$ $0,8 \mathrm{~nm}$ ). Ces ségrégations, imagées en position 1 , cessent de l'être en position 2 (Fig. 2) avec laquelle nous ne sélectionnons que $\mathrm{SiC}_{\beta}$ (111) et $\mathrm{C}(10)$ (Fig. 5).

Nous avons effectué des défocalisations de l'objectif afin d'étudier la structure des ségrégations. La corrélation entre les deux images (Figs. 6 et 7) nous indique que les ségrégations visualisées en position 1 sont microcristallisées. Il en est de même de l'intérieur du fragment.

Ces ségrégations peuvent être formées de carbone turbostratique ou de quartz $\alpha$ (réflexion 101 : $0,334 \mathrm{~nm}$ ).

Nous n'avons pas identifié en diffraction électronique à aire sélectionnée (Fig. 8) de phase pouvant correspondre à du quartz $\alpha$.
Afin de déterminer la nature des microcristallites, nous avons utilisé la technique des franges de réseau. Pour réaliser ces images, nous faisons interférer le faisceau transmis sans déviation et un faisceau diffracté par l'objet. Dans notre cas, nous avons sélectionné la réflexion 002 du carbone turbostratique $\left(\mathrm{C}_{002}\right.$, Fig. 2). Puisque les angles de diffraction sont très petits (environ $0,3^{\circ}$ pour la réflexion $002 \mathrm{du}$ carbone turbostratique $\left(d_{002} \geqslant 0,344 \mathrm{~nm}\right)$, seules les couches carbonées quasi parallèles au faisceau électronique sont imagées. Ces couches sont alors représentées par des franges dans les images. Ceci est illustré sur les figures 9 et 10 . Les figures 11 et 12 représentent respectivement le fond clair (position 0 , Fig. 2) et le fond noir (position 1, Fig. 2) du même fragment. Nous remarquons que la densité des points lumineux en fond noir dans les liserés (Fig. 12) est plus importante que celles des franges en franges de réseau (Fig. 10) pour une même plage (comparer les Figs. 10 et 12). La figure 9 montre le détail d'une petite fraction de la surface externe de la fibre correspondant à la zone fléchée de la figure 11. On constate sur ce cliché que le carbone turbostratique forme une coque.

Il s'ensuit que la phase formant les ségrégations n'est pas uniquement formée de carbone turbostratique. Les microcristallites de carbone sont noyés dans une phase probablement amorphe que nous n'avons pas identifiée.

Les ségrégations constituent des zones de fragilité importantes pour la fibre, leur orientation est unidirectionnelle dans le fragment et les unités qui les composent sont orientées parallèlement à la ségrégation. En effet, si nous tournons le diaphragme objectif de $90^{\circ}$, nous ne constatons pas de ségrégations dans la direction perpendiculaire à la direction primaire (Fig. 13 à comparer à la Fig. 6). Il ne semble pas que ces ségrégations soient liées à la direction d'attaque de la fibre par le couteau de diamant. Il est également peu probable qu'elles soient provoquées par la coupe faite à faible vitesse ( $2 \mathrm{~mm} / \mathrm{min})$.

Quand on forme l'image uniquement avec une partie du faisceau diffracté par le carbure de silicium (position 2, Fig. 2), on constate que l'image est formée de domaines lumineux de taille variable (Fig. 14), les plus importants sont de l'ordre de $5 \mathrm{~nm}$ de diamètre. Nous avons également testé le comportement statistique de la fibre sur cette réflexion. Nous constatons une corrélation entre deux images dont la défocalisation varie de $50 \mathrm{~nm}$ (comparer les Figs. 15 et 16).

Le carbure de silicium se présente donc sous forme de microcristallites dispersés dans une matrice non encore identifiée.

5.2 ETUDE DE LA FIBRE NLP 101. - Cette fibre, contrairement à la précédente, montre très peu de 


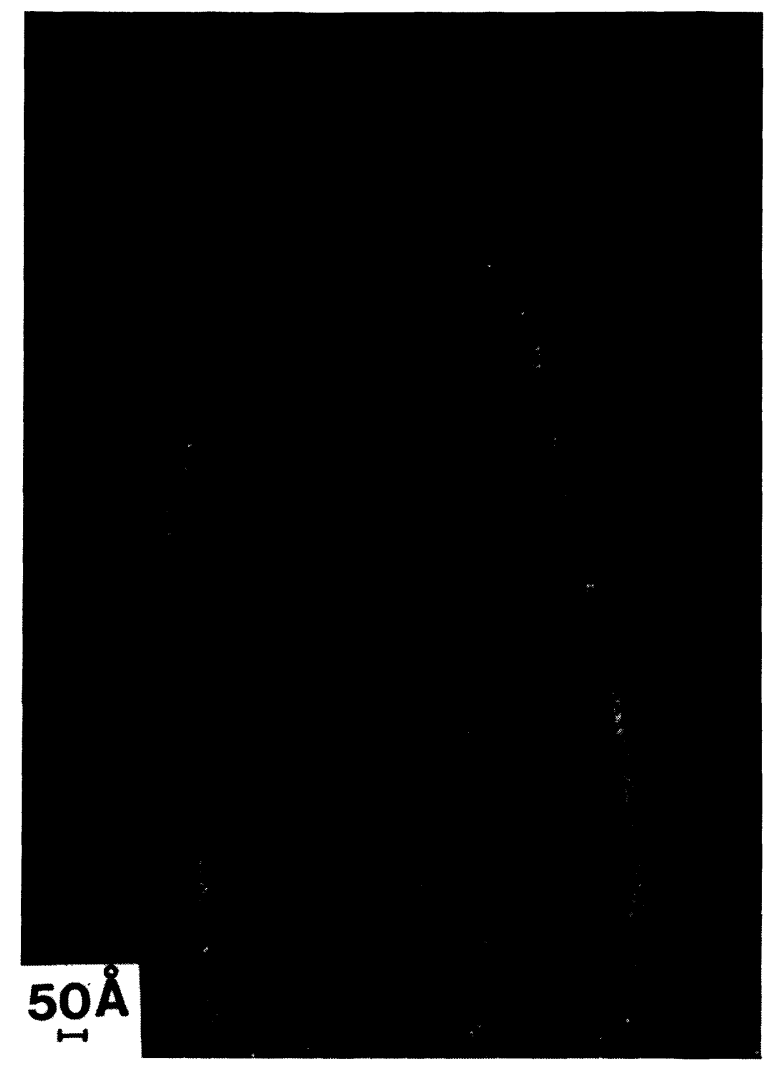

Fig. 4. - Fond noir en position 1 de la fibre NLM 102, $\Delta f=22 \mathrm{~nm}$.

[Dark field image in position 1 NLM 102 fibre, $\Delta f=22 \mathrm{~nm}$.]

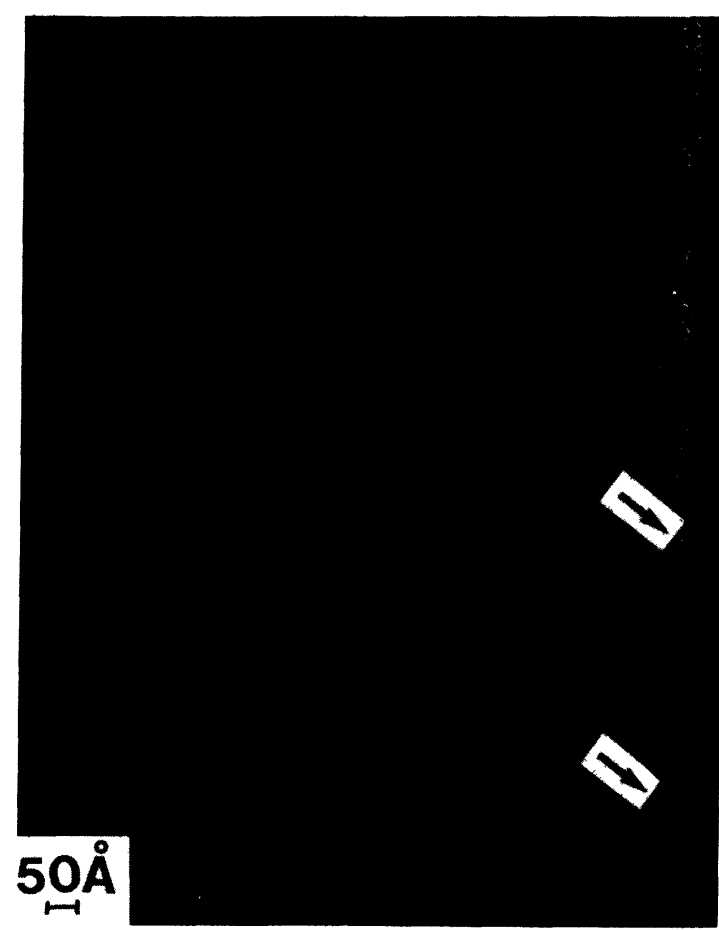

Fig. 6. - Fond noir en position 1 de la fibre NLM 102 ; $\Delta f=22 \mathrm{~nm}$.

[Dark field image in position 1 NLM 102 fibre; $\Delta f=22 \mathrm{~nm}$.]

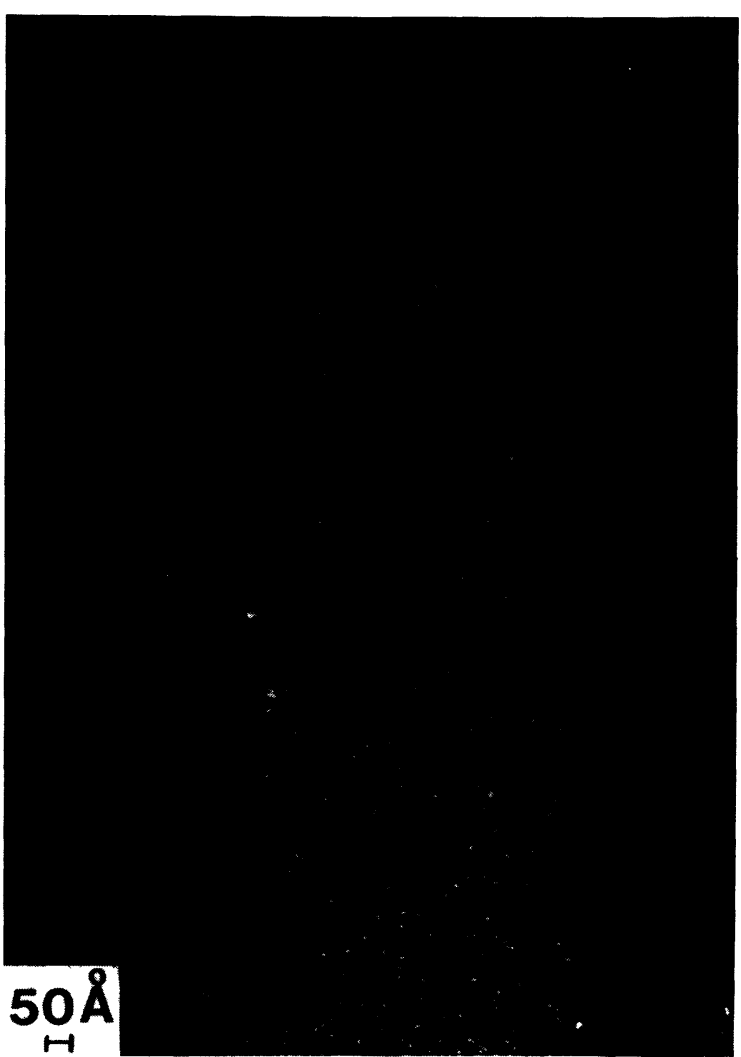

Fig. 5. - Fond noir en position 2 de la fibre NLM 102, $\Delta f=22 \mathrm{~nm}$.

[Dark field image in position 2 NLM 102 fibre, $\Delta f=22 \mathrm{~nm}$.

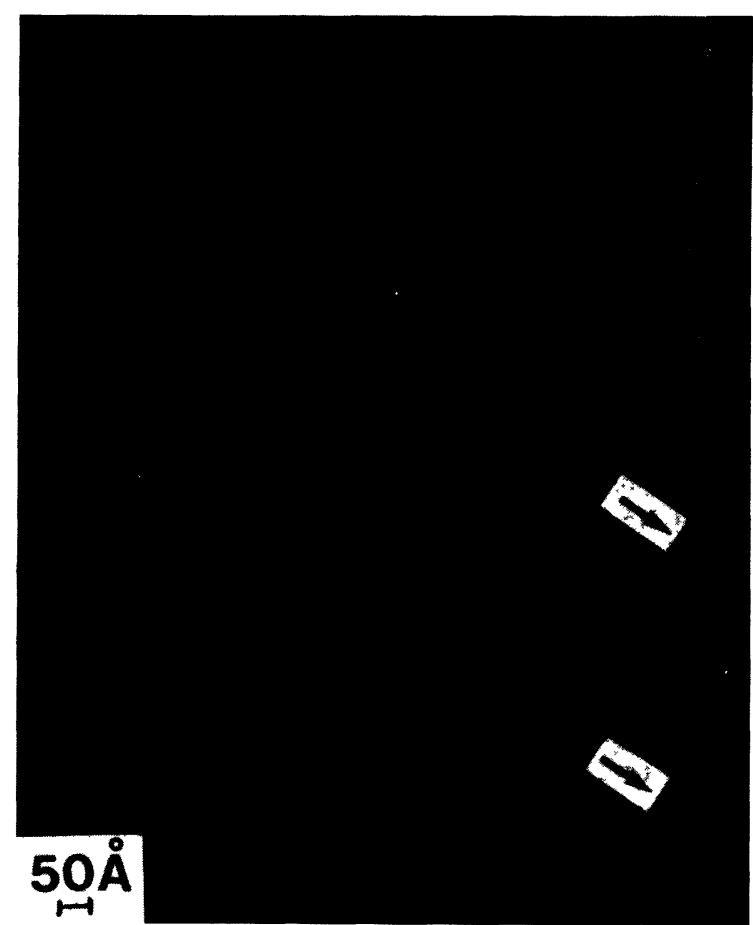

Fig. 7. - Fond noir en position 1 de la fibre NLM 102 ; $\Delta f=72 \mathrm{~nm}$.

[Dark field image in position 1 NLM 102 fibre; $\Delta f=72 \mathrm{~nm}$.] 


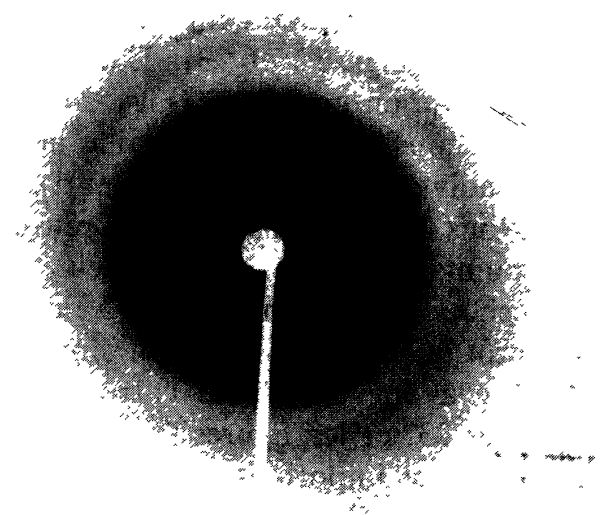

\section{$0.1 \dot{A}^{-1}$}

Fig. 8. - Diffraction électronique à aire sélectionnée de la fibre NLM 102.

[Selected area diffraction pattern, NLM 102 fibre.]

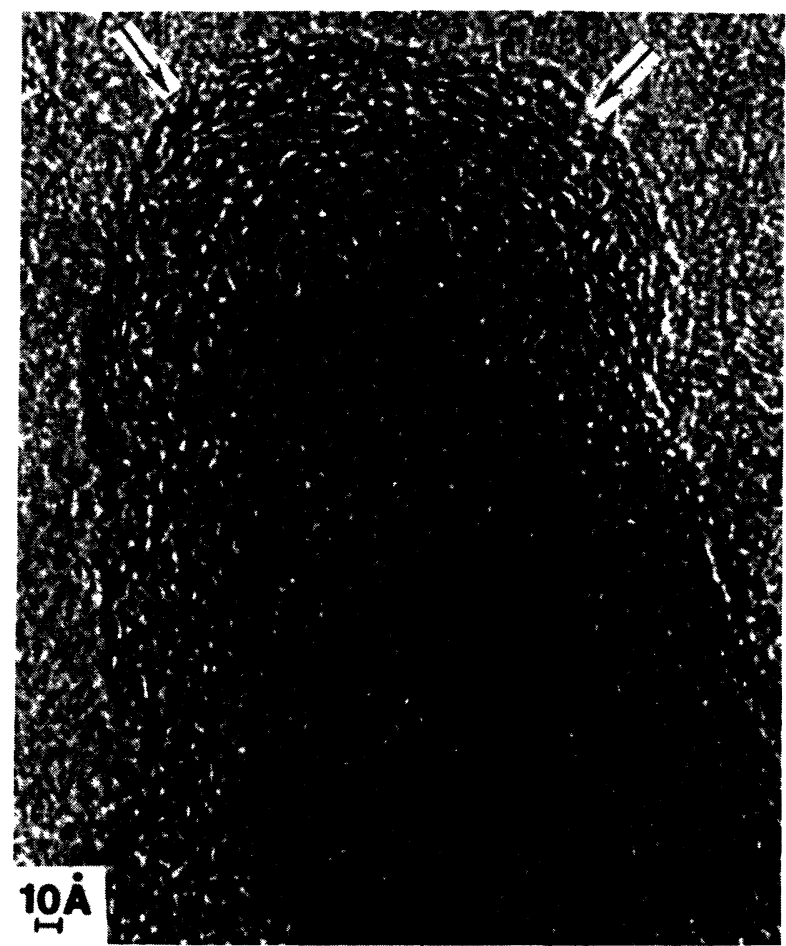

Fig. 9. - Franges de réseau de la fibre NLM 102 dans la région fléchée de la figure 11.

[Lattice fringes image in the region with arrows of the figure 11, NLM 102 fibre.]

fragments avec ségrégations. Les images en fond noir SiC (111) (position 2, Fig. 2) sont constituées d'une distribution homogène de points lumineux.

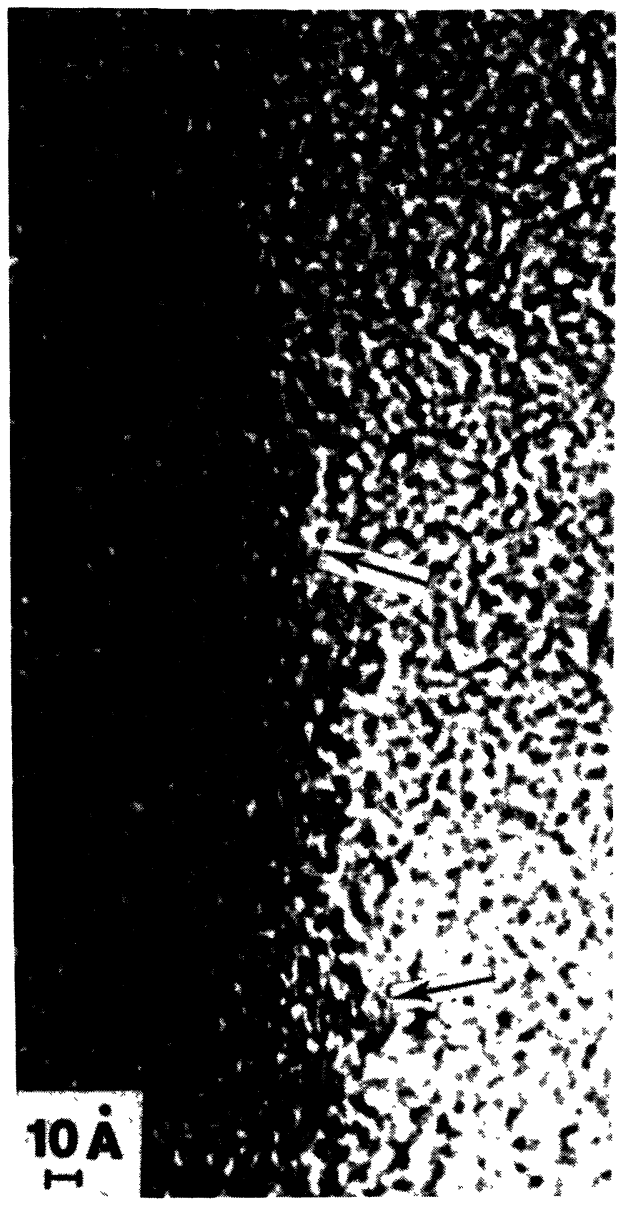

Fig. 10. - Franges de réseau de la fibre NLM 102 dans la région fléchée de la figure 12 .

[Lattice fringes image in the region with arrows of the figure 12, NLM 102 fibre.]

Nous n'avons pas rencontré de microcristallites d'environ $5 \mathrm{~nm}$ de diamètre similaires à ceux présents dans la NLM 102. Nous avons effectué des séries focales $(\Delta f=50 \mathrm{~nm})$ en positions 1 et 2 , de manière à tester le caractère aléatoire ou non de l'image. Il y a une corrélation entre deux images de focalisation différente (Figs. 17 et 18) en position 2 et aucune corrélation en position 1 (Figs. 19 et 20).

La fibre NLP 101 présente donc une phase de carbure de silicium microcristallisée. La taille des microcristallites est plus petite que dans la fibre NLM 102. La phase étudiée en position 1 est amorphe.

5.3 ETUde DE LA FIBRE SIC 1000. - La fibre $\mathrm{SiC} 1000$ présente quelques ségrégations. La fibre est amorphe en position 1 et en position 2, la dimension des points lumineux est d'environ $0,6 \mathrm{~nm}$. Il semble donc qu'elle ait été moins chauffée que les fibres NLM 102 et NLP 101, car elle est moins évoluée structuralement (Fig. 21). 


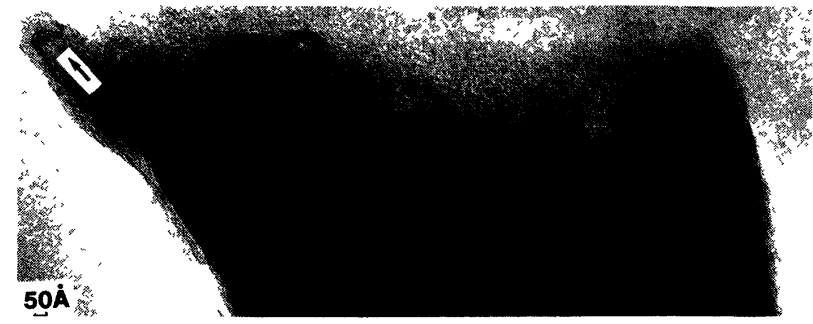

Fig. 11. - Fond clair en position 0 de la fibre NLM 102 (surface de fibre).

[Bright field image in position 0, NLM 102 fibre (fibre surface).]

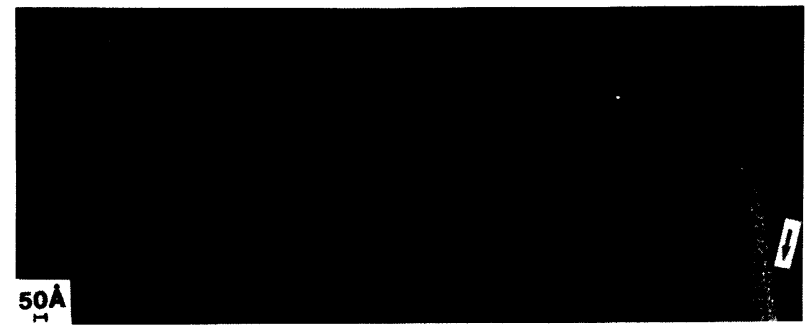

Fig. 12. - Fond noir en position 2 de la fibre NLM 102 (surface de fibre).

[Dark field image in position 2, NLM 102 fibre (fibre surface).]

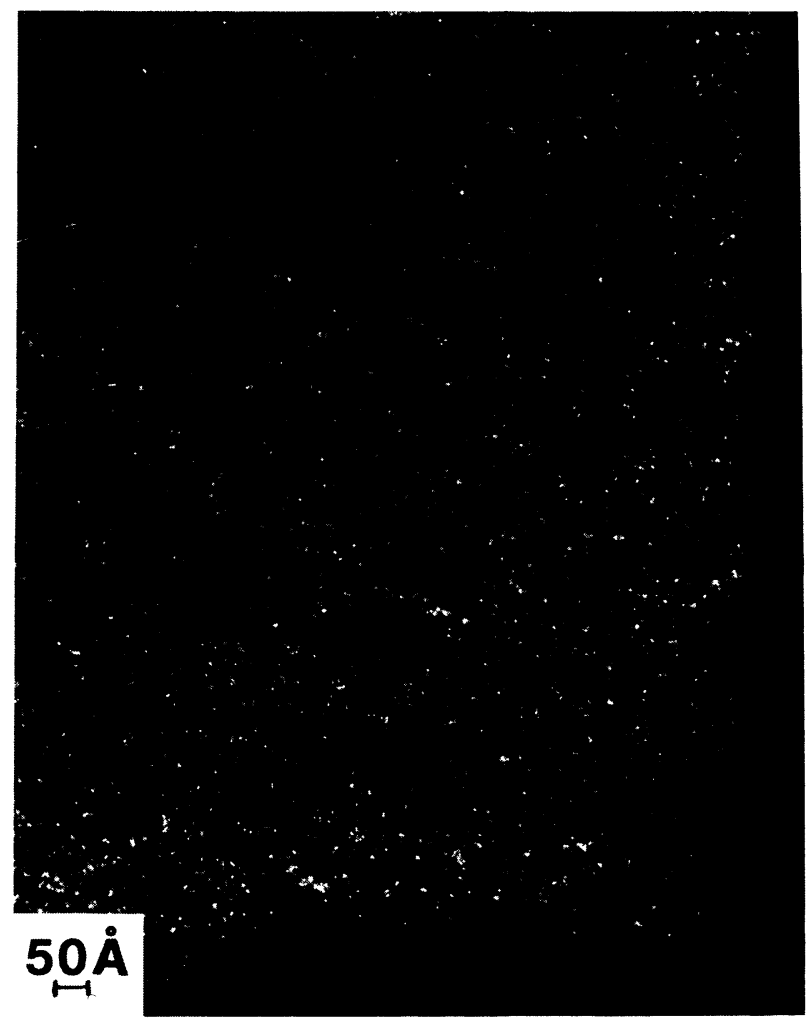

Fig. 13. - Fond noir en position 1 de la fibre NLM 102, $\Delta f=22 \mathrm{~nm}$, à $90^{\circ}$ de la figure 6.

[Dark field image in position 1, NLM 102 fibre, $\Delta f=22 \mathrm{~nm}$, from $90^{\circ}$ of the figure 6.]
5.4 ETUdE DE LA FIBRE NLM 202. - Contrairement aux trois fibres précédemment étudiées, la fibre NLM 202 ne présente pas de ségrégations en position $1 \mathrm{du}$ diaphragme objectif. Elle est microcristallisée en position 1, elle contient donc du carbone turbostratique (Figs. 22 et 23). Elle est également microcristallisée en position 2 (Figs. 24 et 25). Dans ces images, nous notons que la taille des domaines lumineux individuels est homogène et d'environ $0,6-$ $0,8 \mathrm{~nm}$. Nous remarquons de nombreuses ségrégations de points d'environ $2 \mathrm{~nm}$. Chacun d'eux pourrait correspondre à des moirés ou à des franges de Bragg plutôt qu'à une visualisation directe du microcristal. Il y a une excellente corrélation entre les figures 22 et 23 , et les figures 24 et 25 . Le taux de cristallisation est important.

En diffraction électronique, nous ne détectons que la phase $\mathrm{SiC}$ qui est probablement très importante par rapport aux autres phases présentes dans

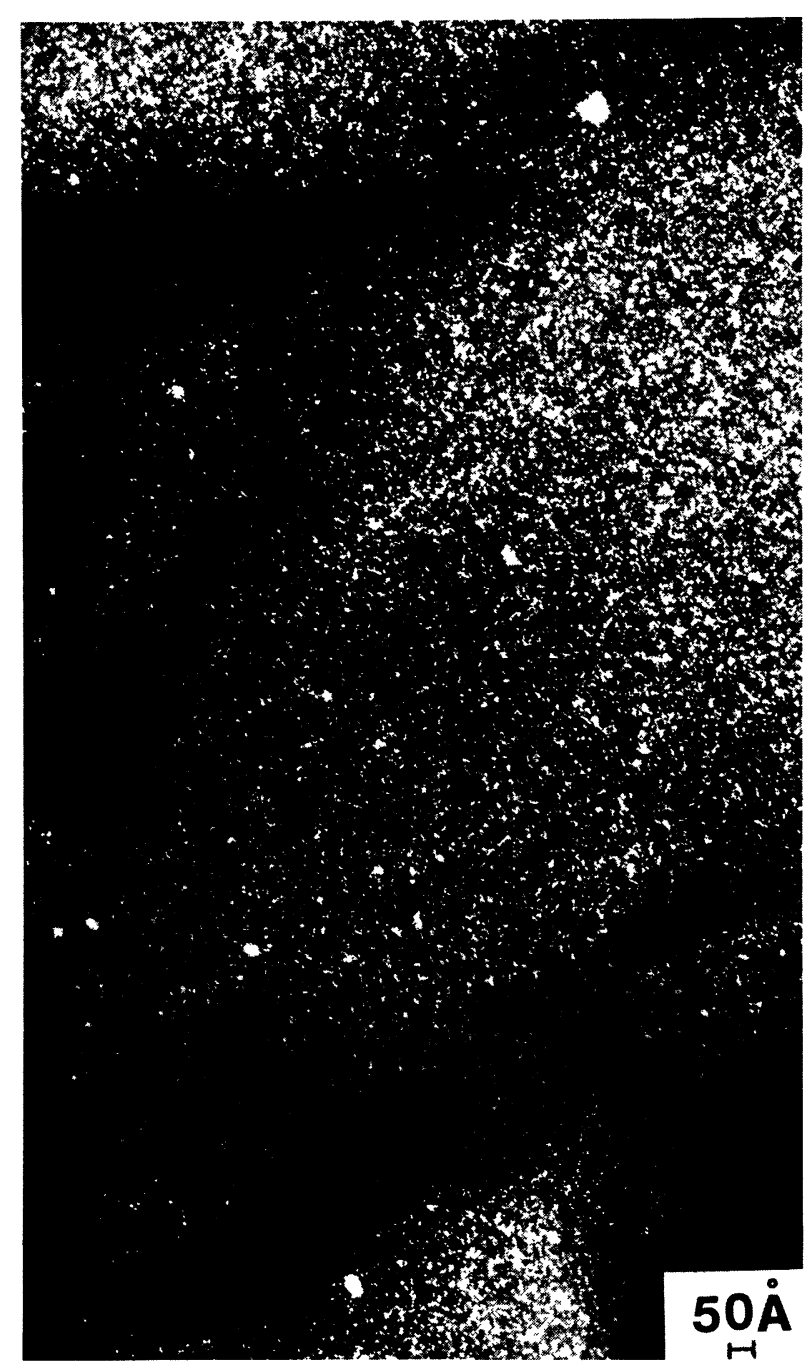

Fig. 14. - Fond noir en position 2 de la fibre NLM 102, $\Delta f=22 \mathrm{~nm}$.

[Dark field image in position 2, NLM 102 fibre, $\Delta f=22 \mathrm{~nm}$. 


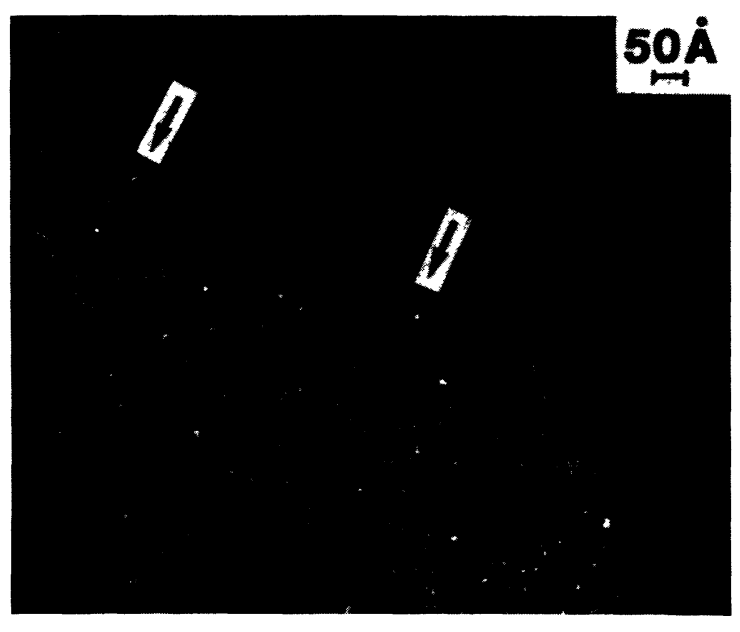

Fig. 15. - Fond noir en position 2 de la fibre NLM 102, $\Delta f=22 \mathrm{~nm}$.

[Dark field image in position 2, NLM 102 fibre, $\Delta f=22 \mathrm{~nm}$.]

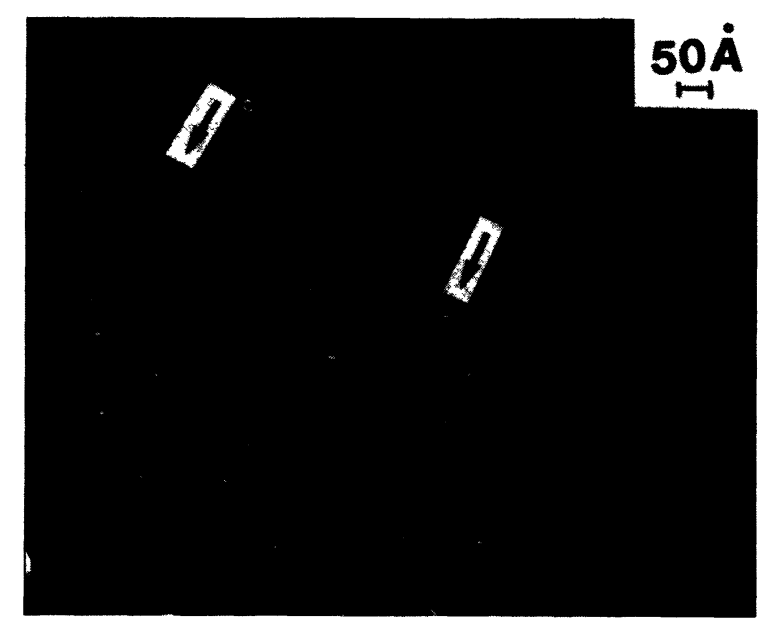

Fig. 16. - Fond noir en position 2 de la fibre NLM 102, $\Delta f=72 \mathrm{~nm}$.

[Dark field image in position 2, NLM 102 fibre, $\Delta f=72 \mathrm{~nm}$.]

cette fibre (Fig. 26). Si nous estimons la taille des microcristallites à partir de la formule de Scherrer, nous trouvons qu'elle est d'environ $3 \mathrm{~nm}$, ce qui ne correspond pas à la taille des points lumineux individuels observés en fond noir. Il est à noter que c'est la taille ultime des microcristallites également trouvée par Simon pour un fluage de la fibre NLP 101 pendant $20 \mathrm{~h}$ à $1300^{\circ} \mathrm{C}$ et pour un recuit des fibres NLP 101 et NLM 102 à $1300^{\circ} \mathrm{C}[6,7,8]$.

\section{Conclusion, discussion.}

- Les ségrégations rencontrées dans les fibres NLP 101 et SiC 1000 sont amorphes, ceux de la fibre NLM 102 sont microcristallisées. Les microdomaines sont du carbone turbostratique.

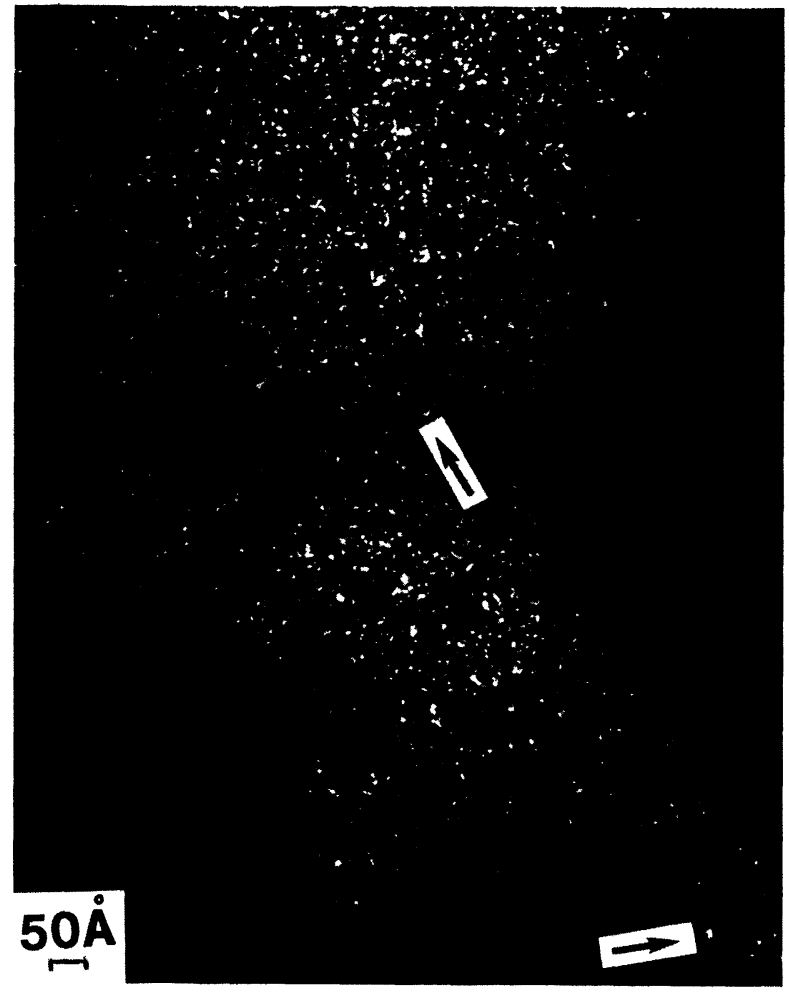

Fig. 17. - Fond noir en position 2 de la fibre NLP 101, $\Delta f=22 \mathrm{~nm}$.

[Dark field image in position 2, NLP 101 fibre, $\Delta f=22 \mathrm{~nm}:]$

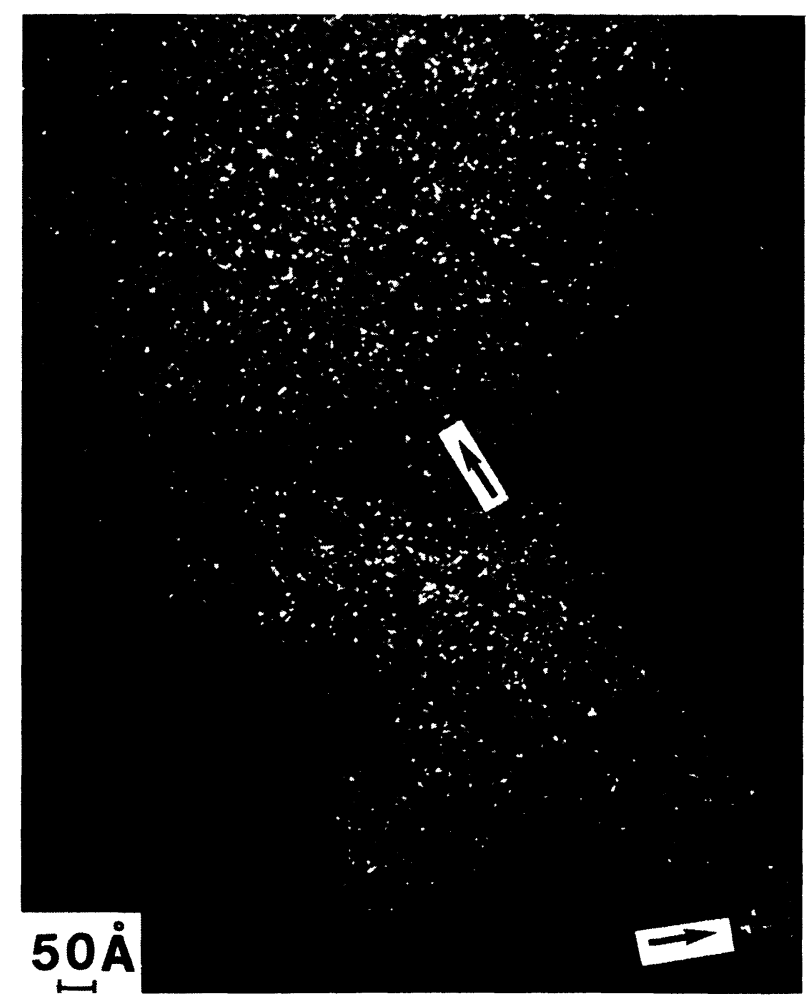

Fig. 18. - Fond noir en position 2 de la fibre NLP 101, $\Delta f=72 \mathrm{~nm}$.

[Dark field image in position 2, NLP 101 fibre, $\Delta f=72 \mathrm{~nm}$.] 


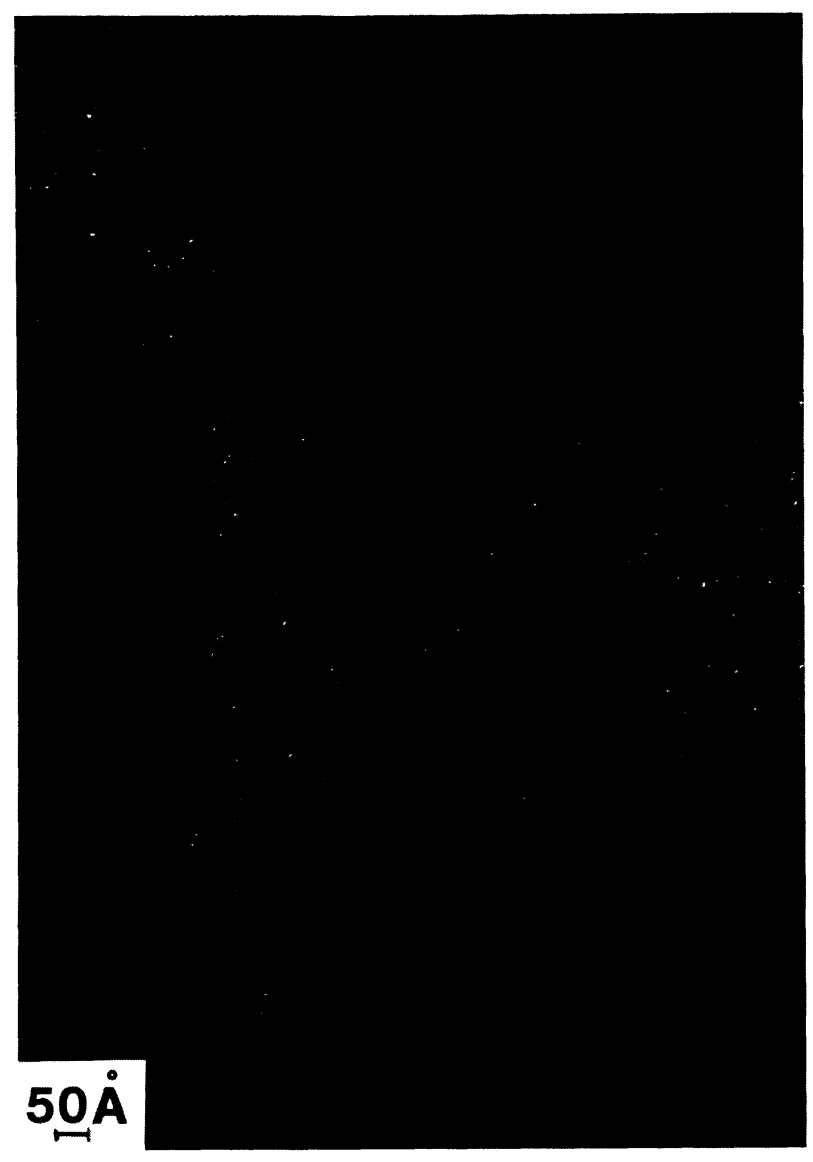

Fig. 19. - Fond noir en position 1 de la fibre NLP 101, $\Delta f=22 \mathrm{~nm}$.

[Dark field image in position 1, NLP 101 fibre, $\Delta f=22 \mathrm{~nm}$.]

- Nous n'avons pas trouvé de ségrégations dans la fibre NLM 202.

- Les fibres NLP 101 et SiC 1000 sont amorphes en position 1, les fibres NLM 102 et NLM 202 contiennent du carbone turbostratique. Les microcristallites de carbone sont noyés dans une phase amorphe que nous n'avons pas identifiée.

- Pour aucune des fibres nous n'avons trouvé de quartz $\alpha$. Par diffraction des rayons $\mathrm{X}$, Simon $e t$ al. $[8,9]$ ont également montré que les fibres NLP 101 et NLM 102 ne contiennent pas de quartz $\alpha$. Remarquons que le quartz a été trouvé par Hasegawa et al. $[4,5]$ à partir de polycarbosilane pyrolysé, mais n'ayant pas été oxydé vers $190^{\circ} \mathrm{C}$. Il est à noter que c'est cette phase de préparation (réticulation) des fibres de carbure de silicium qui permet la formation de liaisons SiOSi et SiOC. L'hypothèse de la présence de la silice a été émise d'après des travaux d'analyse par infrarouge qui ont mis en évidence la vibration de valence de la liaison $\mathrm{SiO}$. Ceci n'implique pas la présence systématique de $\mathrm{SiO}_{2}$ exprimé.

- Toutes les fibres étudiées à l'exception de la fibre $\mathrm{SiC} 1000$ sont partiellement microcristallisées

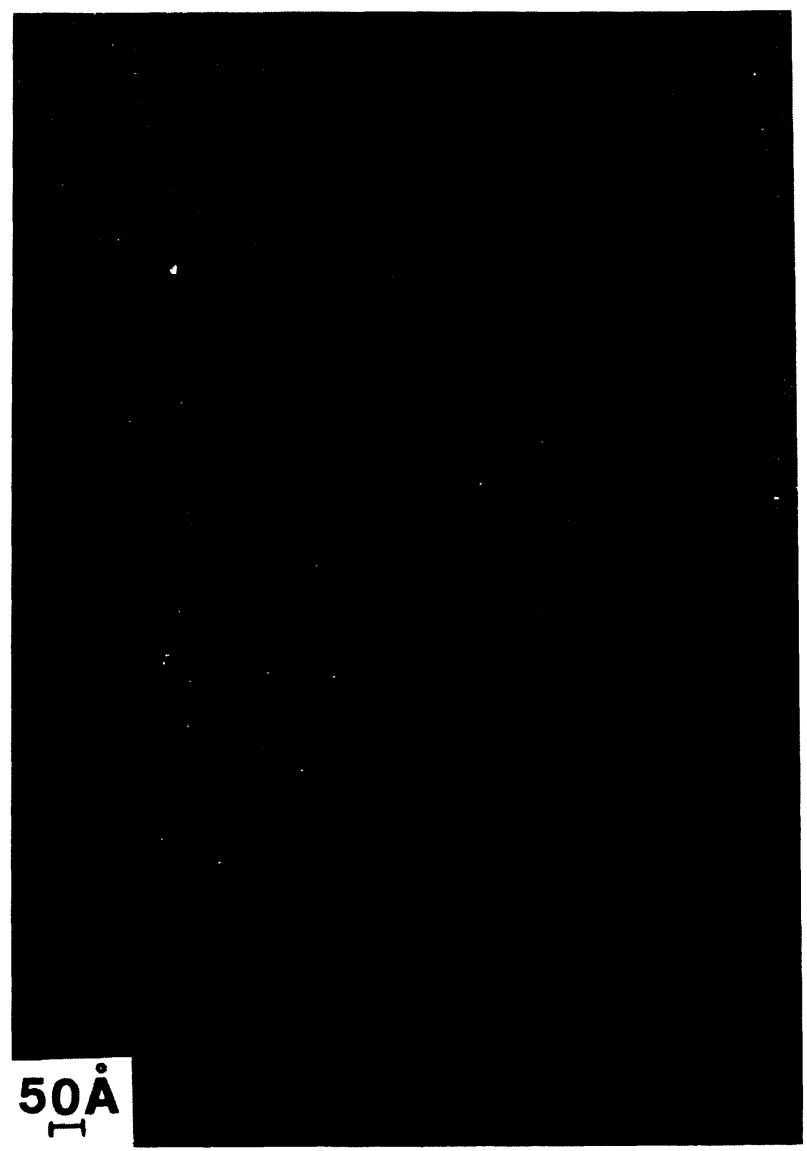

Fig. 20. - Fond noir en position 1 de la fibre NLP 101, $\Delta f=72 \mathrm{~nm}$.

[Dark field image in position 1, NLP 101 fibre, $\Delta f=72 \mathrm{~nm}$.]

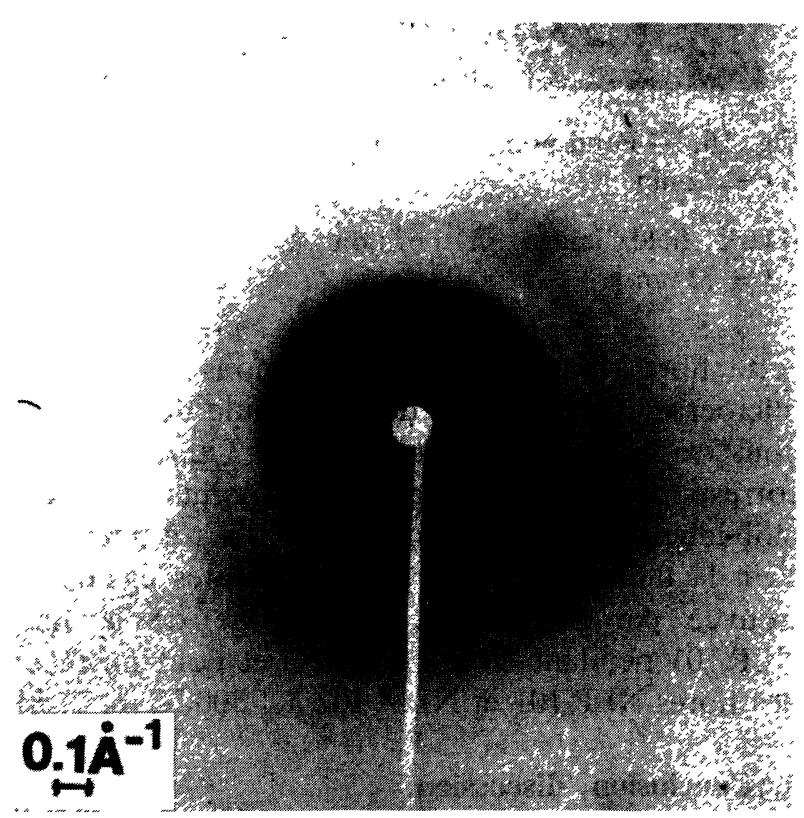

Fig. 21. - Diffraction électronique à aire sélectionnée de la fibre $\mathrm{SiC} 1000$.

[Selected area diffraction pattern, $\mathrm{SiC} 1000$ fibre.] 


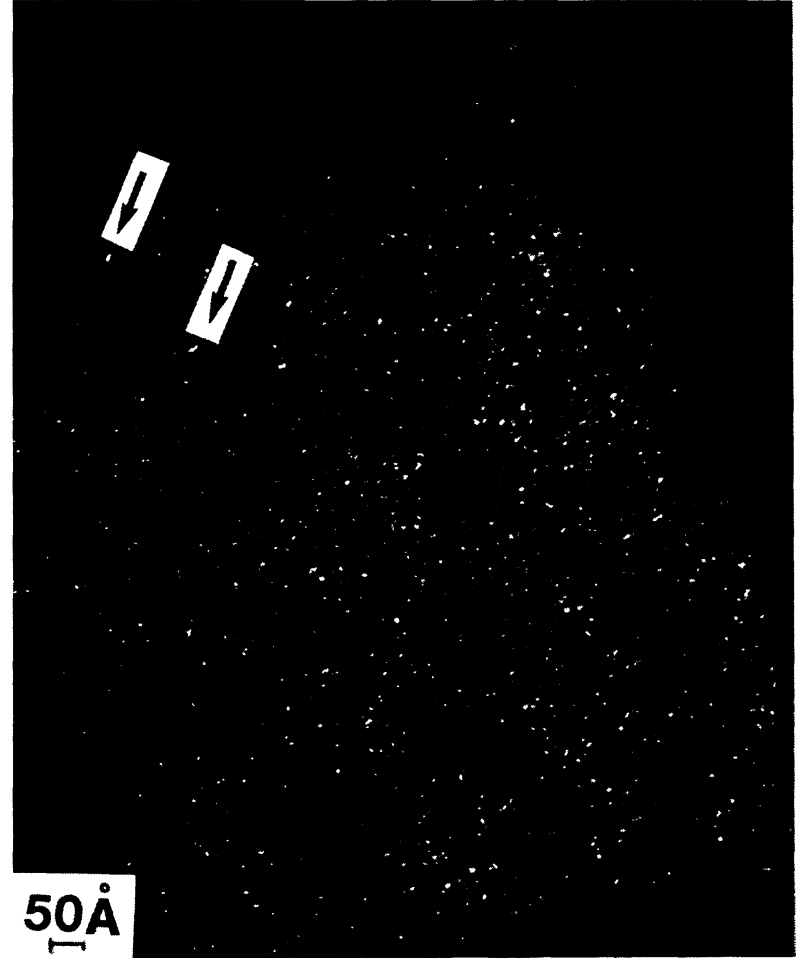

Fig. 22. - Fond noir en position 1 de la fibre NLM 202, $\Delta f=22 \mathrm{~nm}$.

[Dark field image in position 1, NLM 202 fibre, $\Delta f=22 \mathrm{~nm}$.

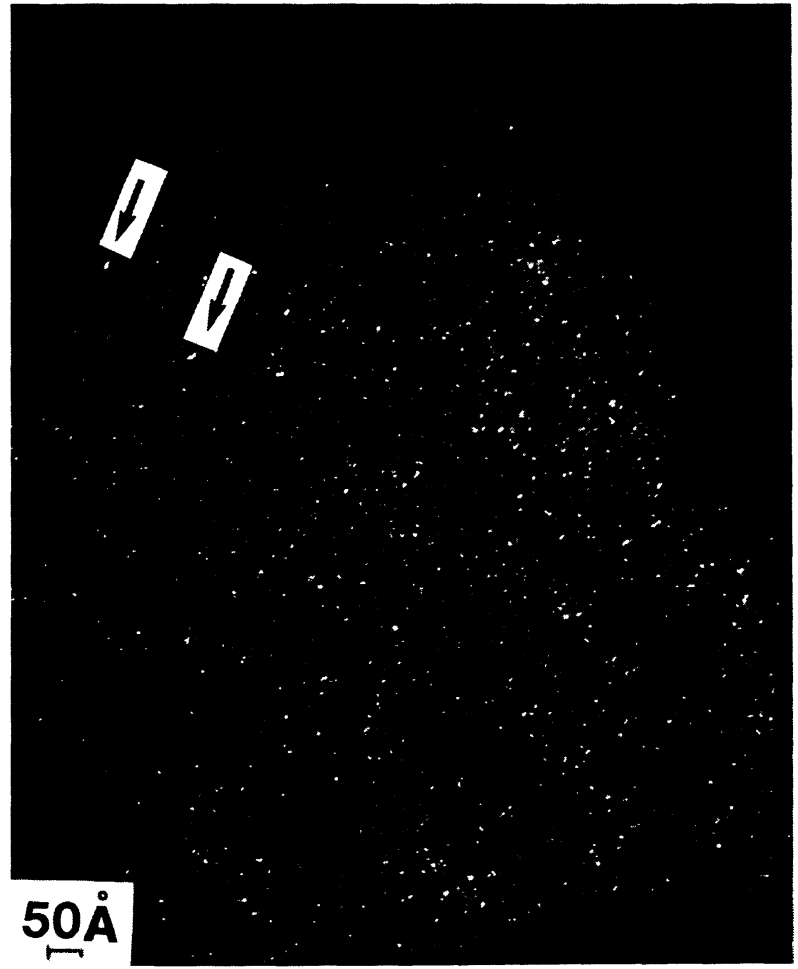

Fig. 23. - Fond noir en position 1 de la fibre NLM 202, $\Delta f=72 \mathrm{~nm}$.

[Dark field image in position 1, NLM 202 fibre $\Delta f=72 \mathrm{~nm}$.

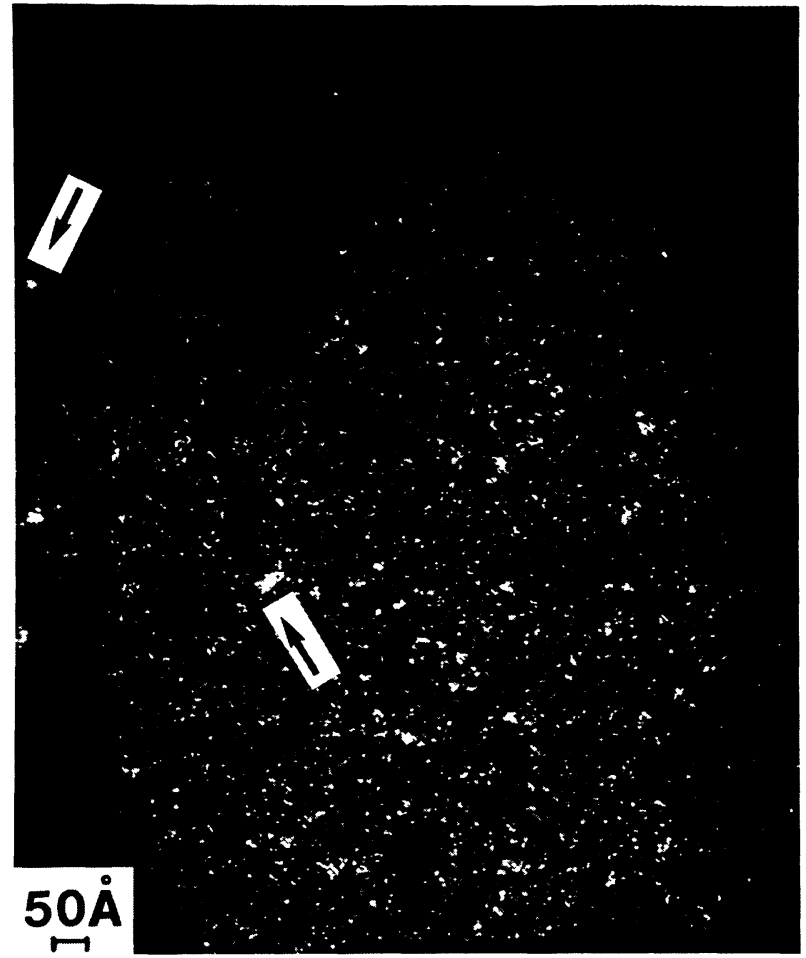

Fig. 24. - Fond noir en position 2 de la fibre NLM 202, $\Delta f=22 \mathrm{~nm}$.

[Dark field image in position 2, NLM 202 fibre, $\Delta f=22 \mathrm{~nm}$.

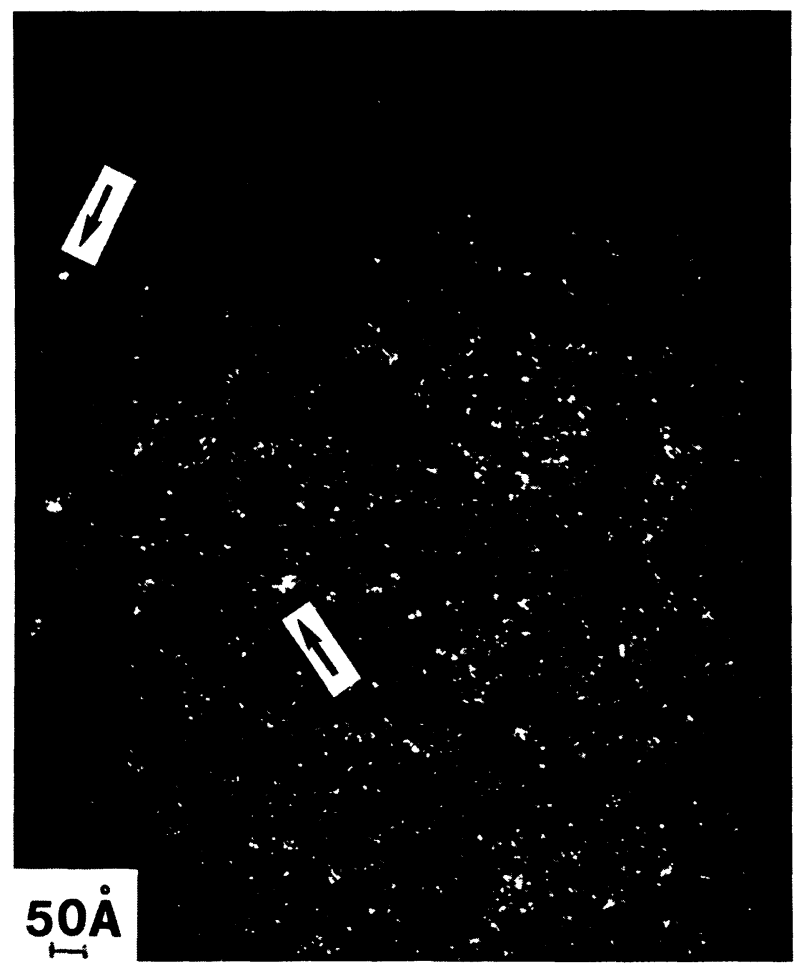

Fig. 25. - Fond noir en position 2 de la fibre NLM 202, $\Delta f=72 \mathrm{~nm}$.

[Dark field image in position 2, NLM 202 fibre, $\Delta f=72 \mathrm{~nm}$.] 


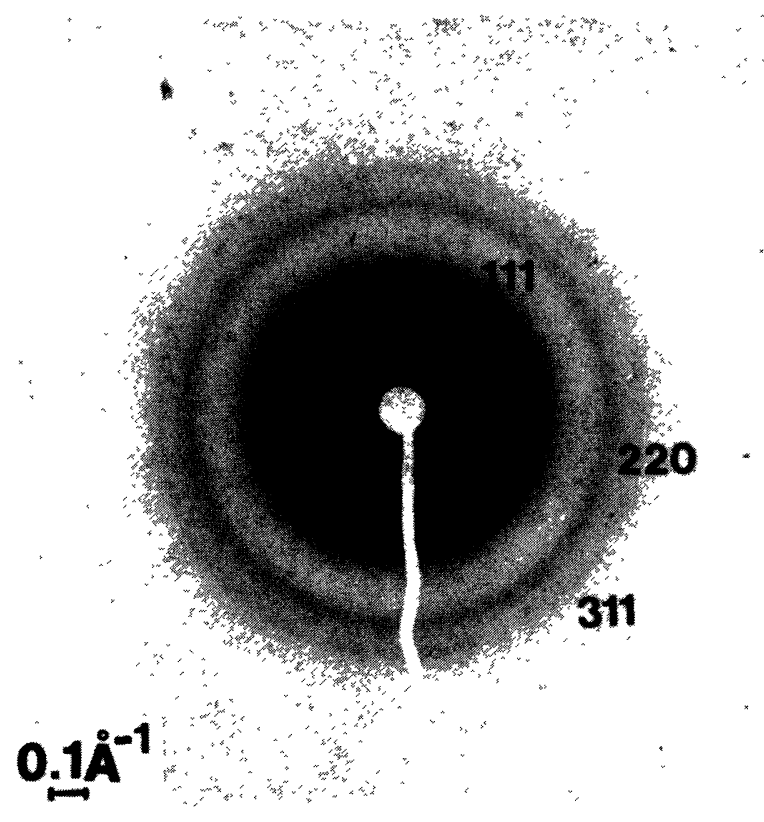

Fig. 26. - Diffraction électronique à aire sélectionnée de la fibre NLM 202.

[Selected area diffraction pattern, NLM 202 fibre.]

en SiC. La cristallinité de la NLM 202 est la plus élevée, mais avec une taille de grains inférieure à celle de la fibre NLM 101. On peut penser que ces caractéristiques (pas de ségrégations de carbone, taille de microcristallites homogène, phase $\mathrm{SiC}$ très importante dans la fibre, fibre homogène) doivent être favorables à de bonnes propriétés mécaniques en traction. En effet, la fibre NLM 102 (carbone et gros grains) a une contrainte en traction égale à $1,49 \mathrm{GPa}$ contre $1,84 \mathrm{GPa}$ pour la fibre NLP 101 (longueur de jauge $25 \mathrm{~mm}[6,7]$ et $2 \mathrm{GPa}$ pour la fibre NLM 202 [9].

- Nous pensons que les fibres NLP 101, SiC 1000 et NLM 102 ont pu être préparées à partir d'un même procédé. Il est probable que les groupements latéraux du type $\mathrm{CH}_{3}$ aient été nombreux dans le précurseur car ce sont ces groupements qui par traitement thermique, conduisent à la formation de carbone en excès.

- La présence de carbone libre dans les fibres a été mise en évidence par spectrométrie Auger par Simon et al. [6-8]. La fibre NLP 101 contient $7 \%$ en poids de carbone tandis que la fibre NLM 102 en contient $15,5 \%$. Nos résultats confirment qualitativement ces données.

- Les différences qui existent entre la fibre NLM 102 et les fibres NLP 101 et SiC 1000 semblent être liées au traitement thermique : la température de ce traitement est plus élevée pour la fibre NLM 102 que pour les deux autres fibres (taille de points lumineux allant jusqu'à $5 \mathrm{~nm}$ contre 0,6 $0,8 \mathrm{~nm}$ pour les autres fibres). Le traitement thermique de ces fibres de carbure de silicium provoquerait une diffusion et une ségrégation du carbone formé pendant la pyrolyse du polycarbosilane puisque les ségrégations de carbone de la fibre NLM 102 sont très importantes et peuvent parfois constituer une coque.

Il semble que les propriétés finales des fibres de carbure de silicium soient étroitement dépendantes des phénomènes qui se produisent lors de leur fabrication avant $500{ }^{\circ} \mathrm{C}$ (oxydation et déshydrogénation du polycarbosilane). Ces phénomènes gouvernent l'état rhéologique du matériau en dessous de $500{ }^{\circ} \mathrm{C}$, qui lui-même impose l'état microtextural, donc les propriétés physiques après pyrolyse. Ces phénomènes sont à rapprocher de ceux qui semblent gouverner les propriétés physiques des fibres de carbone ex-polyacrylonitrile [12].

L'auteur remercie la DRET pour le soutien financier qu'elle a accordé à cette étude, Mme Oberlin pour l'accès au microscope électronique de son laboratoire et Mme Velut pour les coupes à l'ultramicrotome.

\section{Bibliographie}

[1] Oberlin, A., Oberlin, M., Maubois, M., Philos. Mag. 32 (1975) 833.

[2] Chevalier, J. P., Microscopie Electronique en Sciences des Matériaux (CNRS, Paris) 1981.

[3] Yajima, S., Hasegawa, Y., Hayashi, J., IIMURA, M., J. Mater. Sci. 13 (1978) 2569.

[4] Hasegawa, Y., Iimura, M. Yajima, S., J. Mater. Sci. 15 (1980) 720.

[5] Hasegawa, Y., Okamura, K., J. Mater. Sci. 18 (1983) 3633.

[6] Simon, G., Bunsell, A. R., J. Mater. Sci. 19 (1984) 3649.
[7] Simon, G., Bunsell, A. R., J. Mater. Sci. 19 (1984) 3658.

[8] Bunsell, A. R., Simon, G., Comp. Sci. Technol. 27 (1986) 157.

[9] Lesniewski, C., Bunsell, A. R. Rapport de la convention DRET/SEP n 84.445 (1985).

[10] Chaudhari, P., GraczyK, J. F., Herd, S. R., Phys. Status Solid. 51 (1972) 801.

[11] Kakinoki, J., Katada, K., Hanawa, T., Ino, T. Acta Crystallogr. 13 (1960) 171.

[12] Guigon, M., Thèse d'Etat, Compiègne (1985). 